\title{
Intake of Liquid and Solid Sucrose in Relation to Changes in Body Fatness over 6 Years among 8- to 10-Year-Old Children: The European Youth Heart Study
}

\author{
Nanna Julie Olsen ${ }^{a} \quad$ Lars Bo Andersen $^{b, c}$ Niels Wedderkopp ${ }^{b}$ \\ Peter Lund Kristensen $^{\mathrm{b}} \quad$ Berit Lilienthal Heitmann ${ }^{\mathrm{a}, \mathrm{b}}$ \\ ${ }^{a}$ Research Unit for Dietary Studies, Institute of Preventive Medicine, Copenhagen Capital \\ Region, Copenhagen University Hospitals, Frederiksberg, ' University of Southern Denmark, \\ Institute for Sport Sciences and Clinical Biomechanics, Odense, Denmark, ' Norwegian School \\ of Sport Sciences, Department of Sports Medicine, Oslo, Norway
}

\author{
Key Words \\ Soft drinks · Obesity $\cdot$ Children · Insulin
}

\begin{abstract}
Objective: To examine if intake of soft drinks is more closely associated with weight gain than other energy sources and if these associations are mediated through differences in energy intake or insulin level. Methods: Data derived from the Danish part of the European Youth Heart Study $(n=359)$. Height, weight, and waist circumference (WC) were measured in 1997-1998 and again in 2003-2004. Results: Intake of liquid sucrose was associated with changes in waist circumference $(\Delta W C)\left(\beta=0.226, p=0.07, R^{2}=0.17\right)$ as well as BMI z-scores $\left(\Delta \mathrm{BMI}_{\mathrm{z}}\right)\left(\beta=0.031, \mathrm{p}=0.05, \mathrm{R}^{2}=0.30\right)$. Associations attenuated slightly after adjusting for energy intake, but were substantially reduced when adjusting for insulin. Adjustment for both insulin and energy intake attenuated the effect of soft drinks intake on $\Delta \mathrm{BMI}_{\mathrm{Z}}$, but not on $\triangle \mathrm{WC}$. Conclusion: Liquid sucrose seemed more clearly associated with $\triangle \mathrm{WC}$ and $\Delta \mathrm{BMI}_{\mathrm{z}}$ than other energy sources. For $\Delta \mathrm{WC}$, the association seemed to be based on decreases in insulin sensitivity rather than increases in energy intake, whereas for $\Delta \mathrm{BMI}_{\mathrm{z}}$ the association seemed to be based on both increases in energy intake and decreases in insulin sensitivity.
\end{abstract}




\section{Introduction}

Obesity is a serious risk factor for development of diabetes, cardiovascular disease, and certain cancers among adults. However, even among children, obesity is associated with adverse metabolic changes in risk factor levels for these diseases [1-3]. Further, adults who were obese in childhood or adolescence are at an increased risk of cardiovascular disease, atherosclerosis, diabetes, or early mortality [1].

The prevalence of overweight and obesity among school-aged children has increased over the past years [4], and in parallel with the secular trends in obesity, an increase in the availability of soft drinks has been seen in most of the European countries [5].

Four different biological mechanisms have been proposed for an association between intake of liquid sucrose as soft drinks and subsequent changes in body fatness [6,7]. They include:

i) Liquids causes excess caloric intake [6, 7]. Studies have suggested that calories from liquids may bring less satiation than calories from solids. In a review [8], it was concluded that of the four hypotheses, this one has the strongest support, however, the literature was not consistent.

ii) Liquids fail to trigger physiological satiety mechanisms, providing imprecise and incomplete compensation for energy consumed as liquids [9]. Different proposals have been made for this hypothesis, but will not be further introduced here.

iii) Liquids cause lower thermogenesis. This may give rise to a positive energy balance and subsequent weight increase compared to solids [6].

iv) Consumption of sweetened beverages displaces milk intake [8]. This displacement has been found to decrease calcium intake, which may have obesity-lowering properties [8].

We propose a fifth mechanism, e.g. that intake of liquid sucrose and subsequent weight change and obesity development may be mediated through increases in insulin release. Few previous studies have examined the effect of liquid sucrose on insulin level, but one experimental study found a fast (and dramatic) increase in insulin concentration reaching a maximum $1 \mathrm{~h}$ after the consumption [10]. However, to our knowledge no other studies have examined if insulin level may mediate the association between intake of liquid sucrose and changes in body fatness.

The purpose of our study was to examine if soft drinks may be more obesogenic than other energy sources, and if effects of soft drinks on subsequent change in BMI or waist circumference are mediated through differences in energy intake and/or insulin level.

\section{Material and Methods}

Data from the Danish part of European Youth Heart Study (EYHS) was used. EYHS is a prospective study of the associations between lifestyle and risk factors among children and adolescents from Denmark, Portugal, Estonia, and Norway. The ethical procedures operating in each of the four countries were adhered to prior to the commencement of the study. The written consent of the child's parent or legal guardian was obtained [11].

Each school in the municipality of Odense was stratified according to school type. From each stratum, a proportionate two-stage cluster sample of children was selected. Children within 8-10 and 14-16 years of age were allocated code numbers and randomly selected using random number tables. Of 35 schools, 28 were randomly sampled, and 25 (71\%) agreed to participate. Of the 3 non-participating schools, 1 was rural, 1 was urban from middle class area, and 1 was urban from low-income area. All 3 schools gave interference with the educational process as reason for not participating [12].

A total of 589 children ( $37 \%$ of the 3 rd grade population) participated in the baseline examination in 1997-1998 [12]. 
Olsen et al.: Intake of Liquid and Solid Sucrose in Relation to Changes in Body

Fatness over 6 Years among 8- to 10-Year-Old Children: The European Youth Heart Study

The applied study population consisted of 359 children (202 girls and 157 boys) with a mean age of 9.6 years, who had complete information on dietary intake and measurements of BMI and waist circumference at both baseline and the follow-up 6 years later. In each case, where a child or its parents did not participate, the headmaster was asked if the child differed from the rest of the class in any way. Of the children not participating, 1 child was autistic. The remaining children were described as being normal with a normal level of physical activity compared to their class peers [12].

The study design, sampling, and methods have been described in detail elsewhere [11].

Dietary intake was measured using 24-hour dietary recall interview, supplemented by a food frequency questionnaire (FFQ) and a qualitative food record [12].

Fasting insulin level was measured in $\mu \mathrm{IU} / \mathrm{ml}$ blood at serum samples. Samples of fasting insulin were performed using $5 \mathrm{ml}$ vacuumed tubes. After being taken, samples were left to stand at room temperature for $30 \mathrm{~min}$ to allow the serum to clot, and spun for $10 \mathrm{~min}$ before being frozen and stored. The samples were analyzed for fasting insulin level using an enzyme immunoassay.

Information on physical activity was obtained from an FFQ at baseline, and was recoded into a binary variable, applying the questions 'which of these are most you?'. The answers 'I don't exercise' was recoded into 'not physically active'. The answers 'I exercise sometimes, but not regularly' and 'I exercise regularly' were recoded into 'physically active'. Physical activity was also measured using accelerometers. The analyses were performed with both FFQ and accelerometer measurements of physical activity, and the results were essentially similar. However, due to a substantial reduction in the number of participants using accelerometers, it was chosen to present data with measurements of physical activity from the FFQ and use the accelerometer measures in sensitivity analyses.

In the current study, BMI z-score and waist circumference were applied as measures of body fatness to obtain estimates of relative body fatness and abdominal fat. Thus, changes in BMI z-scores $\left(\Delta \mathrm{BMI}_{\mathrm{z}}\right)$ and changes in waist circumference $(\triangle \mathrm{WC})$ were used as outcomes.

Body height to the nearest $5 \mathrm{~mm}$ was measured using a stadiometer. The participants had bare feet or wore stockings. Body weight to the nearest $0.1 \mathrm{~kg}$ was measured using a beam-scale type weight. The participants were lightly dressed. Waist circumference was measured at the largest circumference of the abdomen between the ribs and the trochanter major to the nearest $\mathrm{mm}$. Two measurements of waist circumference were taken, and a mean was calculated.

Cardiorespiratory fitness was defined as maximal power output per kilogram of body weight, and was measured using a graded maximal exercise test on an electronically braked bicycle ergometer (Monark Ergomedic 839, Vansbro, Sweden). For 9-year-olds, initial and incremental workloads were $20 \mathrm{~W}$ for children weighing less than $30 \mathrm{~kg}$, and $25 \mathrm{~W}$ for those weighing $30 \mathrm{~kg}$ or more. Workload was programmed to increase after every $3 \mathrm{~min}$. Heart rate (HR) was recorded continuously (Polar Vantage, Kempele, Finland) throughout the test. The test continued until the child was no longer able to continue. Criteria for maximal effort were i) $H R \geq 185$ and ii) a subjective observation from the researcher that the child could not continue (after vocal encouragement if necessary). The child was considered to have stopped the test if the child's pedaling rate dropped below $30 \mathrm{rpm}$ [11].

\section{Statistical Methods}

BMI z-scores were generated using the LMS method, which summarizes the changing distributions of a covariate by the median, the coefficient of variation and skewness expressed as Box-Cox power [13]. Using z-scores enables comparison of a measured BMI with adequate gender- and age-specific reference values [14]. It was chosen to apply national reference BMI z-scores to the study population. Hence, a power transformation of 0.1 years of age was used [14].

Independent variables included: intake of sugar (all mono- and disaccharides in the diet), intake of sucrose (added sugar), intake of liquid sucrose (carbonated, sugar-sweetened beverage with a sucrose content of $10 \%$ of the volume and sugar-sweetened lemonade, in grams), and intake of solid sucrose ((total intake of added sugars in grams - grams of sucrose from orange juice, apple juice, chocolate milk, milkshake and drinkable yoghurt) - grams of liquid sucrose).

All estimates of associations were per 10-gram intake.

\section{Covariates}

Baseline anthropometrics, intake of complex carbohydrates, intake of fat, physical activity, maternal socioeconomic status, gender, whether puberty started, and specific school were included as potential confounders as well as an interaction term between maternal socioeconomic status and gender. When exam- 
ining intake of liquid sucrose as exposure, the model was adjusted for intake of solid sucrose. When applying intake of solid sucrose as exposure, the model was adjusted for intake of liquid sucrose. The adjustments were done in order to control for possible confounding. The Pearson correlation between intake of liquid sucrose and intake of solid sucrose was not significant (Pearson $R^{2}=0.07, p=0.20$, data not shown).

Total energy intake and fasting insulin level were included separately and together as potential mediators.

All independent and dependent variables were applied as continuous variables in the analyses.

Multiple linear regression analyses were performed in SAS 9.0, using the Proc Mixed procedure. Using the Proc Mixed procedure made it possible to adjust for the cluster sampling. $\mathrm{R}^{2}$ values were found using fixed effects models, using the Proc GLM procedure.

The multiple linear regression analyses were performed in 5 steps:

- $\quad$ Crude model, including the exposure and outcome unadjusted for potential confounders.

- $\quad$ Model 1, including exposure, outcome and potential confounders.

- $\quad$ Model 2, including all covariates in model 1 and total energy intake.

- Model 3, including all covariates in model 1 and fasting insulin level.

- Model 4, including all covariates in model 1 and fasting insulin level and energy intake.

Model 3 and model 4 were examined for potential interaction between intake of soft drinks and fitness level by adding an interaction term to each of the models.

\section{Results}

Table 1 shows the distribution of age, BMI, BMI z-scores, height, weight,waist circumference, total energy intake, intake of sugar, intake of sucrose, intake of liquid sucrose, intake of solid sucrose, fasting insulin level, and physical fitness by gender at baseline. A significant difference between boys and girls was observed in age $(p=0.01)$, height $(p=0.02)$, total energy intake $(p=0.01)$, total intake of sugar $(p=0.01)$, total intake of added sugar $(p=0.02)$, total intake of liquid sucrose $(\mathrm{p}=0.01)$, fasting insulin level $(\mathrm{p}=0.03)$, and physical fitness $(p<0.0001)$ when t-test analyses were performed (data not shown). For all other covariates, no significant differences between boys and girls were observed (data not shown).

Total intake of sugar was not associated with changes in body fatness (table 2), but a borderline significant association between intake of sucrose and $\triangle \mathrm{WC}$ was found, also after adjusting for baseline anthropometrics and other potential confounders (table 2). Intake of sucrose was not associated with $\Delta \mathrm{BMI}_{\mathrm{z}}$, neither before nor after adjustment for baseline anthropometrics and other confounders (table 2).

Intake of liquid sucrose was significantly associated with $\Delta \mathrm{WC}$ in the crude model, and borderline significantly associated with $\Delta \mathrm{WC}$ after adjusting for baseline anthropometrics and confounders (crude model and model 1, table 2). Compared to model 1, the estimate weakened slightly when adjusting for total energy intake (model 2, table 2). When adjusting for insulin level (model 3, table 2), the estimate weakened substantially compared to the estimate in model 1. Adjustment for both insulin and energy intake gave essentially similar results (model 4, table 2 ).

Intake of liquid sucrose was significantly associated with $\Delta \mathrm{BMI}_{\mathrm{z}}$ after adjusting for baseline anthropometrics and confounders (model 1, table 2). When further adjusting for total energy intake (model 2, table 2), the estimate weakened slightly compared to model 1 , and the association became non-significant. When adjusting for insulin level, the estimate was substantially weakened compared to model 1 (model 3, table 2). Further adjustment for both energy intake and insulin level weakened the estimate further (model 4, table 2).

Total energy intake and fasting insulin level were not correlated (Pearson $R^{2}=0.03$, $\mathrm{p}=0.55$, data not shown).

There was no interaction between intake of liquid sucrose and physical fitness in any of the models (all $p$ values $\geq 0.29$, data not shown). 
Table 1. Baseline distribution of age, BMI, BMI z-score, height, weight, waist circumference, total energy intake, intake of sugar, intake of sucrose, intake of liquid sucrose, intake of solid sucrose, fasting insulin level, and physical fitness by gender $(\mathrm{n}=359$ unless stated otherwise)

\begin{tabular}{|c|c|c|c|c|c|c|c|c|}
\hline & \multicolumn{4}{|c|}{ Girls $(n=202)$} & \multicolumn{4}{|c|}{ Boys $(n=157)$} \\
\hline & mean & sd & $\min$ & $\max$ & mean & sd & $\min$ & $\max$ \\
\hline Age, years & 9.6 & 0.4 & 8.6 & 10.5 & 9.7 & 0.4 & 8.7 & 10.8 \\
\hline BMI, $\mathrm{kg} / \mathrm{m}^{2}$ & 17.2 & 2.5 & 12.6 & 27.3 & 17.1 & 2.0 & 13.5 & 25.1 \\
\hline BMI z-score, SD & 0.4 & 1.1 & -2.8 & 3.1 & 0.4 & 1.0 & -2.1 & 3.4 \\
\hline Height, cm & 138.2 & 6.5 & 119.4 & 156.0 & 139.8 & 6.2 & 124.1 & 158.8 \\
\hline Weight, kg & 33.1 & 6.2 & 18.4 & 56.5 & 33.7 & 5.8 & 23.2 & 54.5 \\
\hline Waist circumference, $\mathrm{cm}$ & 58 & 5.7 & 47.5 & 84.5 & 58.5 & 5.1 & 49 & 79.8 \\
\hline Total energy intake, MJ & 8.8 & 2.1 & 3.8 & 15.5 & 9.5 & 2.5 & 3.7 & 20.6 \\
\hline Total intake sugar, g & 103.5 & 40.8 & 22.6 & 267.1 & 115.0 & 45.9 & 22.4 & 259.8 \\
\hline Total intake sucrose, g & 48.6 & 34.0 & 0 & 182.3 & 57.4 & 39.9 & 0 & 243.8 \\
\hline Intake of liquid sucrose, $\mathrm{g}$ & 18.0 & 22.6 & 0 & 122.5 & 24.6 & 27.8 & 0 & 160.3 \\
\hline Intake of solid sucrose, $g$ & 28.3 & 22.5 & 0 & 123.7 & 31.2 & 131.4 & 0 & 137.3 \\
\hline Fasting insulin level, $\mu \mathrm{IU} / \mathrm{ml}^{\mathrm{a}}$ & 51.5 & 26.1 & 8.1 & 137.6 & 45.4 & 22.1 & 9.3 & 135.2 \\
\hline Physical fitness, $\mathrm{W} / \mathrm{kg}^{\mathrm{b}}$ & 2.9 & 0.5 & 1.6 & 4.2 & 3.3 & 0.5 & 2.0 & 4.5 \\
\hline
\end{tabular}

${ }^{\mathrm{a}}$ Girls $(\mathrm{n}=177)$, boys $(\mathrm{n}=145) .{ }^{\mathrm{b}}$ Girls $(\mathrm{n}=186)$, boys $(\mathrm{n}=142)$.

Table 2. Linear regression analyses of associations between intake of sugar, intake of sucrose, intake of liquid sucrose, and intake of solid sucrose and changes in body fatness over 6 years $(n=359$ unless stated otherwise)

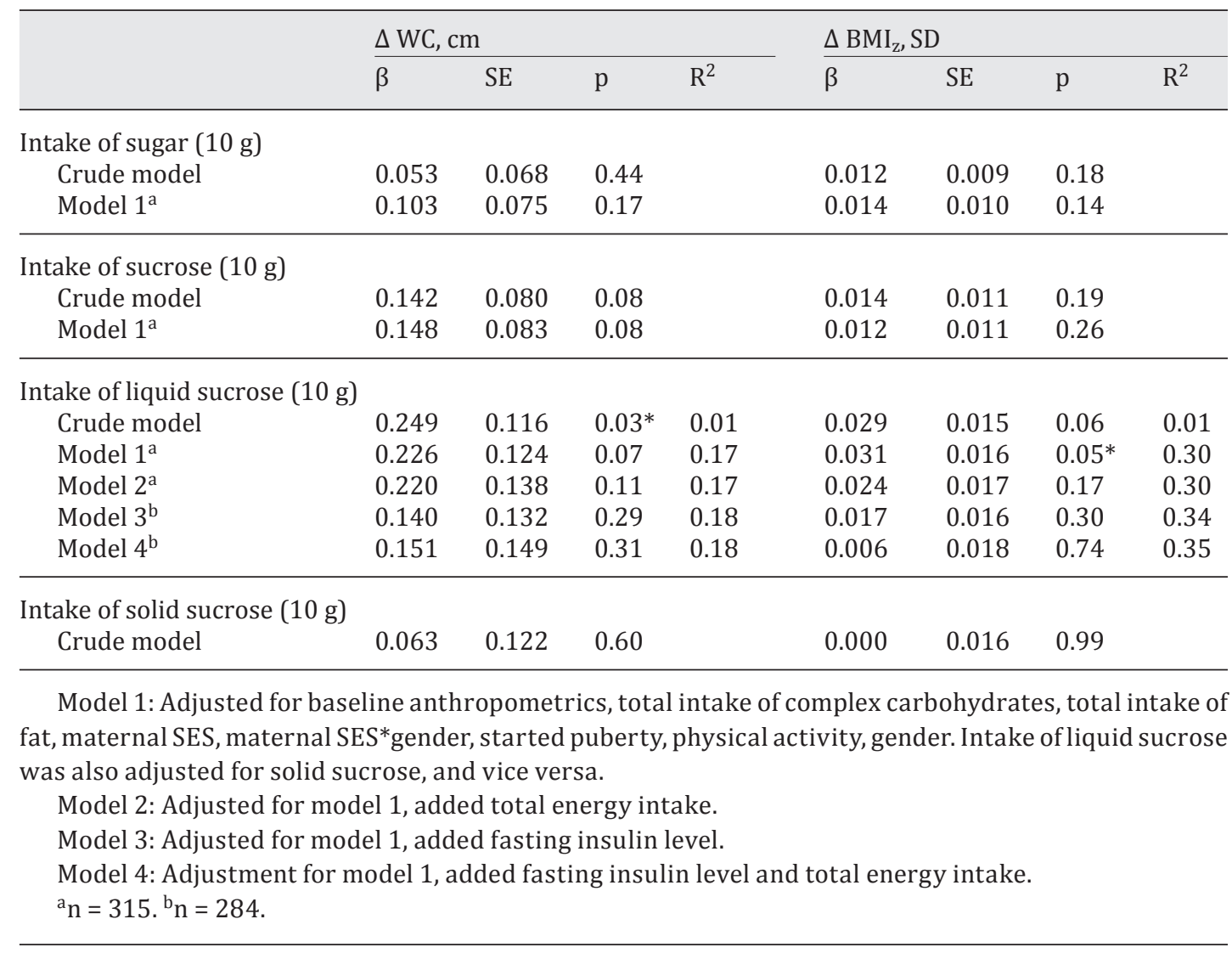


Intake of solid sucrose was not associated with $\Delta \mathrm{WC}$ or with $\Delta \mathrm{BMI}_{\mathrm{z}}$ (table 2).

On a subset of 228 individuals, accelerometer measurements of physical activity were available. Using this variable rather than the more crude measure of physical activity as a binary variable to measure physical activity gave essentially similar results, however, all estimates were non-significant (data not shown). This is probably due to a decreased power when using these measurements.

\section{Discussion}

As hypothesized, we found that liquid sucrose was more closely associated with changes in BMI z-scores and waist circumference than sugar, sucrose, and solid sucrose. We also found that this association was mediated through both total energy intake and fasting insulin level, but that fasting insulin level seemed to be a stronger mediator of the association than total energy intake when examining changes in waist circumference. This is interesting, as the prevailing hypothesis disclosures that differences in total energy intake are biologically linking liquid sucrose to changes in body fatness. However, the present results suggest that insulin sensitivity may play a particular role for mediating effects of soft drink intake on obesity development and in particular abdominal fat development, and that the more rapid rise in insulin levels after consumption of liquid than solid sucrose [10] may offset the subsequent gain in weight, potentially since the increase in insulin concentration may cause increased fatty acid synthesis. Moreover, the quick increases in insulin concentration may cause insulin resistance, due to decreased insulin sensitivity induced by postprandial hyperglycemia [3], which may be especially linked to fat located viscerally instead of subcutaneously as suggested by the results of the present study [15]. No prior studies have examined if fasting insulin level mediate the effects of liquid sucrose on subsequent weight changes, and additional studies examining the role of insulin on the association between liquid sucrose and changes in body fatness are needed before a firm conclusion can be drawn. Furthermore, as far as known, this study is the first to examine if liquid sucrose is associated with changes in fat distribution. Our results are interesting since not many determinants of changes in waist circumference have been identified previously. One longitudinal study found refined bread to be associated with changes in waist circumference in women; but, besides this, only few studies on determinants for changes in waist circumference has been repeated [16].

It may be speculated that mediation of the effects of liquid sucrose on obesity development via insulin may be modified by level of fitness, e.g. that persons with a high fitness may gain less body weight despite large soft drink consumption, while persons with low fitness will increase weight more with high soft drink intake. However, in the present study, we did not find support for this, as there were no interactions between physical fitness level and intake of liquid sucrose in relation to obesity or fat distribution development.

A few limitations should be noted. First, it may be argued that in response to consuming sucrose, oral glucose tolerance test (OGTT) may express insulin release better than fasting insulin level. However, fasting insulin level was considered to be a proxy for OGTT in the present study, relying on the fact that many previous studies have found that fasting insulin level, or the homeostatic model assessment (HOMA) provides an estimate for insulin sensitivity in individuals with an intact $\beta$-cell production, and is closely associated with fitness level [17-20].

Second, the observed associations between intake of liquid sucrose and $\Delta \mathrm{BMI}_{\mathrm{z}}$ and $\Delta \mathrm{WC}$ may be considered weak. However, table 1 shows that the average daily intake of liquid sucrose was $18.0 \mathrm{~g}$ for girls and $24.6 \mathrm{~g}$ for boys. The estimated effects are per 10 grams of intake, suggesting that for an average intake of liquid sucrose the estimated effects would be approximately doubled. The variation in intake of liquid sucrose was large for both 
genders, with some having a daily intake 6 times higher than the mean. It may therefore be assumed that there are some subgroups of children with a very high intake of soft drinks that may be at particularly high risk of developing overweight and obesity. Due to the enlargement of soft drink bottles that has taken place over the last years, it may be assumed that the average daily intake of liquid sucrose would have risen today compared to baseline in 1996-1997. Indeed, other data on dietary habits of Danish children have shown that the average daily intake of soft drinks has risen to $32 \mathrm{~g}$ of liquid sucrose in 2001 [21]. This may suggest that the exposure level has increased over the last years, and hence that intake of soft drinks has become a more important determinant of obesity than previously.

Third, it is possible that residual confounding has been present, particularly in relation to physical activity, due to a crude categorizing. However, when we conducted the analyses using physical activity measured with accelerometers, we obtained essentially similar results, even if, due to the reduction of children with complete data from accelerometry, the associations were not significant.

Finally, reporting bias in relation to sugar consumption may have taken place. However, so far only a few determinants of underreporting among children have been identified. One study found that obese children reported an energy intake $25 \%$ lower than their estimated energy expenditure and that the tendency to underreport increased with age of the child [22]. Furthermore, girls have been found to underreport more than boys in some studies [22], but a similar degree of underreporting has also been found [23]. One study among adults found that obese tended to particularly underreport food items high in carbohydrates [24]. This may also include liquid sucrose. If this assumption is true, the association between intake of liquid sucrose and changes in body fatness observed in the present study will have been underestimated, rather than inflated.

Finally, due to the method used to provide the variable liquid sucrose, food items such as ice cream, soluble chocolate powder, and energy drinks were included in the exposure solid sucrose. It may be discussed if these items would have been more correctly classified as liquids. Thus, it cannot be excluded that the classification of solid sucrose may have caused some underestimation of the associations. On the other hand, the fact that we, despite the potential measurement error mentioned above, still found significant associations again speaks in favor of a true association.

\section{Conclusion}

Intake of sugar, sucrose, and solid sucrose was not associated with 6-year changes in body fatness in this study. On the other hand, intake of liquid sucrose was associated with 6-year changes in waist circumference and BMI z-scores. Our results suggest that a high intake of soft drinks may lead to both abdominal and overall obesity. For abdominal obesity, the association seemed to be based on decreases in insulin sensitivity rather than increases in energy intake, whereas for overall obesity the association seemed to be based on both increases in energy intake and decreases in insulin sensitivity.

\section{Acknowledgements}

We thankfully acknowledge Danish Heart Foundation, Danish Medical Research Council, Health Foundation, Danish Council for Sports Research, Foundation of 17-12-1981, Foundation in Memory of Asta Florida Bolding nee Andersen, Faculty of Health Sciences, University of Southern Denmark and the Tryg Foundation for providing financial support for this study. We also thankfully acknowledge all of the participating children in EYHS and Claus Holst for providing statistical help. 


\section{Disclosure Statement}

None disclosed.

\section{References}

1 Dietz WH: Health consequences of obesity in youth: childhood predictors of adult disease. Pediatrics 1998; 101:518-525.

2 Ebbeling CB, Pawlak DB, Ludwig DS: Childhood obesity: public-health crisis, common sense cure. Lancet 2002;360:473-482.

- 3 Larsson SC, Bergkvist L, Wolk A: Consumption of sugar and sugar-sweetened foods and the risk of pancreatic cancer in a prospective study. Am J Clin Nutr 2006;84:1171-1176.

- 4 Lobstein T, Baur L, Uauy R, for the IASO International Obesity Task Force: Obesity in children and young people: a crisis in public health. Obes Rev2004;5Suppl 1):4-85.

- 5 Naska A, Bountziouka V, Trichopoulou A: Soft drinks: time trends and correlates in twenty-four European countries. A cross-national study using the DAFNE (Data Food Networking) databank. Public Health Nutr 2010;13:1346-1355.

6 Mølgaard C, Andersen NL, Barkholt V, Grunnet N, Hermansen K, Nyvad B, Pedersen BK, Raben A, Stender S: Influence of Sugar on Health (in Danish). The Danish Nutrition Council, 2003

- 7 Bawa S: The role of the consumption of beverages in the obesity epidemic. JR Soc Promot Health 2005;125: 124-128.

8 Bachman CM, Baranowski T, Nicklas TA: Is there an association between sweetened beverages and adiposity? Nutr Rev 2006;64:153-174.

- 9 Almiron-Roig E, Chen Y, Drewnowski A: Liquid calories and the failure of satiety: how good is the evidence? Obes Rev 2003;4:201-212.

10 Janssens JP, Shapira N, Debeuf P, Michiels L, Putman R, Bruckers L, Renard D, Molenberghs G: Effects of soft drink and table beer consumption on insulin response in normal teenagers and carbohydrate drink in youngsters. Eur J Cancer Prev 1999;8:289-295.

11 Riddoch C, Edwards D, Page A, Froberg C, Anderssen SA, Wedderkopp N, Brage S, Cooper AR, Sardinha LB, Haaro M, Heggeboe LK, van Mechelen W, Boreham C, Ekelund U, Andersen LB; the European Youth Heart Study team: The European Youth Heart Study - cardiovascular disease risk factors in children: rationale, aims, study design, and validation of methods. J PhysActiv Health 2005;2:115-129.

-12 Andersen LB, Wedderkopp N, Hansen HS, Cooper AR, Froberg K: Biological cardiovascular risk factors cluster in Danish children and adolescents: the European Youth Heart Study. Prev Med 2003;37:363-367.

-13 Cole TJ, Green PJ: Smoothing reference centile curves: the LMS method and penalized likelihood. Stat Med 1992;11:1305-1319.

14 Nysom K, Mølgaard C, Hutchings B, Fleischer Michaelsen K: Body mass index of 0 to 45-y-old Danes: reference values and comparison with published European reference values. Int J Obes 2001;25:177-184.

15 Hilsted J, Borch-Johnsen K, Christiansen JS: Diabetes. Copenhagen, Munksgaard, 2007.

-16 Halkjaer J, Sorensen TI, Tjonneland A, Togo P, Holst C, Heitmann BL: Food and drinking patterns as predictors of 6-year BMI-adjusted changes in waist circumference. Br J Nutr 2004;92:735-748.

-17 Andersen LB, Boreham CA, Young IS, Davey SG, Gallagher AM, Murray L, McCarron P: Insulin sensitivity and clustering of coronary heart disease risk factors in young adults. The Northern Ireland Young Hearts Study. Prev Med 2006;42:73-77.

18 Wallace TM, Matthews DR: The assessment of insulin resistance in man. Diabet Med 2002;19:527-534.

19 Wallace TM, Levy JC, Matthews DR: Use and abuse of HOMA modeling. Diabetes Care 2004;27:1487-1495.

20 Matthews DR, Hosker JP, Rudenski AS, Naylor BA, Treacher DF, Turner RC: Homeostasis model assessment: insulin resistance and beta-cell function from fasting plasma glucose and insulin concentrations in man. Diabetologia 1985;28:412-419.

21 Fagt S, Matthiessen J, Biltoft-Jensen A, Groth MV, Christensen T, Hinsch HJ, Hartkopp H, Trolle E, Lyhne N Møller A: The development of the Danish people's diet 1985-2001. With focus on sugar and alcohol plus motivation and barriers for a healthy lifestyle (in Danish). The Danish Institute for Food and Veterinary Research. Department for Human Nutrition, 2004.

22 Livingstone MB, Black AE: Markers of the validity of reported energy intake. J Nutr 2003;133(suppl 3):895S-920S.

23 Champagne CM, Baker NB, DeLany JP, Harsha DW, Bray GA: Assessment of energy intake underreporting by doubly labeled water and observations on reported nutrient intakes in children. J Am Diet Assoc 1998; 98:426-433.

24 Heitmann BL, Lissner L: Dietary underreporting by obese individuals - is it specific or non-specific? BMJ 1995;311:986-989. 